Simmel Studies

\title{
Die Geburt der Lebensphilosophie aus dem Geist der Konfliktsoziologie
}

\section{Gregor Fitzi}

Volume 20, numéro 1-2, 2016

URI : https://id.erudit.org/iderudit/1040121ar

DOI : https://doi.org/10.7202/1040121ar

Aller au sommaire du numéro

\section{Éditeur(s)}

Georg Simmel Gesellschaft

\section{ISSN}

1616-2552 (imprimé)

2512-1022 (numérique)

Découvrir la revue

\section{Citer cet article}

Fitzi, G. (2016). Die Geburt der Lebensphilosophie aus dem Geist der Konfliktsoziologie. Simmel Studies, 20(1-2), 137-153.

https://doi.org/10.7202/1040121ar

\section{Résumé de l'article}

The development of Simmel's "life philosophy" characterizing the late phase of his work is ordinarily presented as the product of the reception of his contemporaries, above all Henri Bergson, whom he contributed to translate and introduce in Germany. The present essay shows, however, that Simmel's life-forms paradigm has a completely different origin. It emerges from the essential core of Simmel's theory of modern society, i.e. from his theory of social conflict. By showing that conflict is not simply a dividing factor but a process of sociation that integrates societies, Simmel realized that the epistemological dichotomy between process and substance had to be overcome in and open-ended conception of their dialectics going beyond Hegel's teleological conception of historical development. Simmel found the semantics to express his conception in the examination of the life philosophy of his time, but he radically transformed it to point out the problem he wanted to address. To understand the particular nature of his question, it is important to trace its origins within his sociology. 


\section{ABHANDLUNGEN}

Gregor Fitzi

\section{Die Geburt der Lebensphilosophie aus dem Geist der Konfliktsoziologie}

\section{Verflüssigung und Verdichtung}

Bergsons Wirkung auf die deutsche Reflexion des frühen 20. Jahrhunderts - besonders als Theoretiker der erlebten Zeitlichkeit - ist kaum zu überschätzen. Trotzdem überragt sein Ruhm im literarischen Milieu bei Weitem den in akademischen Kreisen ${ }^{1}$. Das metaphysische Problem des Bewusstseins geht laut Bergson aus dem Konflikt des unaufhaltbaren, mannigfachen Flusses des inneren Zeitbewusstseins mit der räumlichen Struktur des Handelns hervor. Sie prägt die Sprache als gesellschaftliche Institution. So lässt sich die durée als existentielle Tiefschicht nicht erfassen,

${ }^{1}$ Dies gilt bsw. für Stefan George und Ernst Gundolf, die sich Anfang Februar 1909 Bergsons Werke besorgten. Dazu vgl. Seekamp, H. -J.; Ockenden, R. C.; Keilson, M. (1972), Stefan George / Leben und Werk. Eine Zeittafel, Amsterdam, Castrum Peregrini Presse, S. 196. Im Mai 1909 wird über Georges Meinung bezüglich Bergsons [in der George verpflichteten Kleinschreibung] berichtet: "George gibt ihm soweit er es beurteilen kann eine ganz hervorragende bedeutung und meint dass vieles von seiner ahnung über die weiterentwicklung des denkens sich hier bestätigt findet und dass hier über alle 'deutsche philosophie' hinaus ein blick des denkens in die neuen lande geworfen wird die die neue dichtung erschlossen hat", ebd., S. 199. Vgl. auch Meyer, Rudolf W. (1982), Bergson in Deutschland. Unter besonderer Berücksichtigung seiner Zeitauffassung, in «Phänomenologische Forschungen», Bd. 13, Studien zum Zeitproblem in der Philosophie des 20. Jahrhunderts, S. 10-64, S. 18. 
solange sie hinter den räumlichen Formen der Sprache verborgen bleibt ${ }^{2}$. Dank einer "seismographischen Sensibilität" für die Kulturströmungen der Zeit gehört Simmel zu den ersten, die in Deutschland auf Bergson aufmerksam werden. Der beschleunigte Zyklus der monetären Masse in der modernen Geldwirtschaft treibt die Verflüssigung der sozialen Beziehungen voran und drück den Menschen ein stets schneller werdendes Tempo des Lebens auf ${ }^{3}$. Der Vergesellschaftungsrhythmus spiegelt sich im zugespitzten Gegensatz zwischen Statik und Dynamik der Erfahrung wider. Auf die Inflation der sinnlichen Eindrücke reagieren die Neurasthenie und die Blasiertheit großstädtischen Geisteslebens ${ }^{4}$. Im Zuge des Säkularisierungsprozesses tritt keine "ethische Kultur" an die Stelle der religiösen Orientierung, sondern die sich rapide durchsetzende Diktatur des Lebensstils ${ }^{5}$. Als Mode untersteht dieser dem Imperativ der steten Erneuerung. Ein Zeitalter des Werterelativismus bahnt sich an. Anstelle der soliden sozialen Beziehungen, des herkömmlichen Gemeinschaftslebens, treten die momentanen Kollisionen der im fiebrigen Lebensrhythmus der Großstadt rein formell vergesellschafteten Individuen. Der Mensch zieht sich zunehmend zurück und lässt die sozialen Institutionen, vor allem das Geld, zwischen ihm und dem Mitmenschen vermitteln. Die Individualität fragmentiert sich, indem sie sich fortschreitend ausdifferenziert ${ }^{6}$. Einzelne Persönlichkeitsfacetten, und nicht die ganze Person, bedienen unterschiedliche soziale Beziehungen. So verschiebt sich der inhaltliche Schwerpunkt des Lebens von der qualitativen

${ }^{2}$ Bergson, Henri (1889), Essai sur les données immédiates de la conscience, Paris, PUF, 19976, hier zit. mit ED. Dt. Ders. (1920), Zeit und Freiheit. Eine Abhandlung über die unmittelbaren Bewußtseinstatsachen, Berechtigte Übersetzung, Jena, Diederichs, 1920, hier zit. mit ZF.

${ }^{3}$ Simmel Georg (1897), Die Bedeutung des Geldes für das Tempo des Lebens; jetzt in Ders. (1997), Georg Simmel Gesamtausgabe, hier mit GSG zitiert, Bd. 5, Aufsätze und Abhandlungen 1894-1900, Frankfurt/M, Suhrkamp, S. 215-234.

${ }^{4}$ Simmel, Georg (1903), Die Großstädte und das Geistesleben; jetzt in GSG 7, Frankfurt/M, Suhrkamp, 1995, S. 116-131.

${ }^{5}$ Simmel, Georg (1902), Tendencies in German Life and Thought since 1870; jetzt in GSG 18, Englischsprachige Veröffentlichungen 1893 - 1910, hrsg. v. David P. Frisby, Frankfurt am Main, Suhrkamp, 2008, S. 167-202.

${ }^{6}$ Simmel Georg (1890), Über sociale Differenzierung. Soziologische und psychologische Untersuchungen; jetzt in GSG 2, Frankfurt/M, Suhrkamp, 1989, S. 109-295, bes. 169-198. 
zur quantitativen Individualität ${ }^{7}$. Alles befindet sich im Fluss, alles ist ersetzbar, alles lässt sich umgestalten. Doch der Schein trügt.

Wie in der Wirtschaft, so finden auch in der Kultur Akkumulationsprozesse statt. Die Produkte der gesellschaftlichen Arbeit verdichten sich zu Institutionen; die Erzeugnisse der schöpferischen Tätigkeit zum Moloch der “objektiven Kultur", mit dem die "subjektive Kultur" der Individuen nicht mithalten $\mathrm{kann}^{8}$. Ausdifferenzierte soziale Wertsphären wie Politik, Wirtschaft, Wissenschaft, Religion, Kunst und Erotik verselbständigen sich zu autonomen Gebilden mit eigener Geltungslogik ${ }^{9}$. Indem die Kommunikationsmedien verlangen, bedient und fortgesetzt $\mathrm{zu}$ werden, entziehen sie den Menschen die schöpferische Energie. Die rigide Logik der Institutionen überlagert die bewegliche Struktur des menschlichen Denkens und Handelns. Ihre Beziehung kann sich nur noch als Konflikt zwischen Leben und Formen gestalten ${ }^{10}$.

Wissenssoziologisch betrachtet, steht der pessimistische Umschwung in der Selbstwahrnehmung der Moderne im Zusammenhang mit den andauernden Krisen und Rezessionen der Jahrhundertwende, die der wirtschaftlichen Expansionsphase der Gründerzeit folgen ${ }^{11}$. Politisch hängt die Stimmung mit dem rasanten Altern der Machtstrukturen des Kaiserreichs als "Obrigkeitsstaat" zusammen ${ }^{12}$. An der kulturgeschichtlichen Schwelle von der impressionistischen zur expressionistischen Malerei taucht auch eine erste deutsche Übersetzung von Bergsons "neuer Philosophie" auf. Es ist 1908. Bergson soll als Ehrengast zum Dritten Internationalen Philosophiekongress nach Heidelberg kommen, den Xavier Léon mit Wilhelm Windelband

7 Simmel, Georg, Die beiden Formen des Individualismus, in GSG 7, Frankfurt/M, Suhrkamp, 1995, S. 49-56.

${ }^{8}$ Simmel, Georg, Persönliche und sachliche Kultur, in: GSG 5, Frankfurt/M, Suhrkamp, 1992, S. 560-582.

${ }^{9}$ Vgl. Weber, Max $\left(1920^{1}\right)$, Zwischenbetrachtung, in: Ders. (1988), Gesammelte Aufsätze zur Religionssoziologie. Bd. 1, Tübingen, J. C. B. Mohr (Paul Siebeck), S. 536-573.

10 Simmel Georg (1918), Der Konflikt der modernen Kultur, in GSG 16, Frankfurt/M, Suhrkamp, 1999, S. 181-207.

11 Vgl. dazu Dahme, Heinz-Jürgen (1988), Der Verlust des Fortschrittsglaubens und die Verwissenschaftlichung der Soziologie. Ein Vergleich von G. Simmel, F. Tönnies und M. Weber, in Rammstedt O. (Hrsg.) (1988), Simmel und die frühen Soziologen. Nähe und Distanz zu Durkheim, Tönnies und Max Weber, Frankfurt/M, Suhrkamp, S. 222-274.

${ }^{12}$ Weber, Max (1918), Parlament und Regierung im neugeordneten Deutschland; jetzt in Ders., Gesammelte politische Schriften (1921 ${ }^{1}$ ). 5. Aufl. Hrsg. v. Johannes Winckelmann, Tübingen, J. C. B. Mohr (Paul Siebeck), 1988, S. 306-443, bes. S. 333 ff. 
organisiert $^{13}$. Er kann dem Kongress jedoch nicht beiwohnen. Sein Dauererschöpfungszustand hindert ihn daran. Trotzdem schlägt Bergsons Philosophie wie ein Blitz in die philosophische Landschaft der belle époque ein. Dies übersieht Simmel nicht, der aus ganz anderen Gründen nicht nach Heidelberg kommt ${ }^{14}$. Bergsons Aufwertung der erlebten Zeitlichkeit scheint der Dauerfrustration moderner Individualität einen metaphysischen Ausweg zu bieten. Im Konflikt der individuellen mit den kollektiven Zielsetzungen spiegelt sich die Krise der Moderne wider, die nun eine Letztbegründung in der widersprüchlichen Struktur der menschlichen Lebenserfahrung bekommt. Durée und Räumlichkeit. Musik und Sprache. Intimität und Gesellschaft. Den Gegensatz vom Philosophen aufgetischt zu bekommen, der als Absolvent der École Normale Supérieure in der modernen Mathematik und Physik zu Hause ist, beflügelt die schöpferische Ader von Denkern und Literaten. So greift jeder, der auch nur ein bisschen Französisch kann, zu Bergsons Büchern und besonders zur gerade erschienenen Évolution créatrice zurück. Die anderen begnügen sich mit der seit 1908 lieferbaren deutschen Übersetzung von Matière et mémoire ${ }^{15}$. Dort trifft sie allerdings die bittere Enttäuschung.

Isaak Benrubi, der in Griechenland geborene und in Bulgarien aufgewachsene Übersetzer, ist als Weltbürger in Europa zuhause und spricht sowohl Französisch als auch Deutsch. Als Max Scheler, mit dem Vorstoß kommt, Bergsons Philosophie in Deutschland einzuführen, beauftragt Rudolf Eucken Benrubi, der bei ihm über Rousseau promoviert, mit der BergsonÜbersetzung ${ }^{16}$. Beim Erscheinen von Materie und Gedächtnis kam es

${ }^{13} \mathrm{Vgl}$. Bericht über den III. Internationalen Kongress für Philosophie zu Heidelberg, Nendeln/Liechtenstein, Klaus Reprint, 1974.

${ }^{14}$ Dazu vgl. Buch des Dankes an Georg Simmel. Briefe, Erinnerungen, Bibliographie, (1958), hrsg. v. Kurt Gassen und Michael Landmann, Berlin: Duncker und Humblot, S. 24-28.

${ }^{15}$ Bergson, Henri (1896), Matière et mémoire. Essai sur la relation du corps à l'esprit, Alcan, Paris, jetzt PUF, Paris 19934' dt. Bergson, Henri (1908), Materie und Gedächtnis. Essays zur Beziehung zwischen Körper und Geist, erste Auflage, mit einer Einleitung von Wilhelm Windelband, Diederichs, Jena. Von der Übersetzung verspracht sich der Verleger Eugen Diederichs, politisches Kapital für seine konservativ-esoterische Weltanschauung zu schlagen. Vgl. dazu Pflug, Günther (1990), Eugen Diederichs und Henri Bergson, in: Von Göschen bis Rowohlt. Beiträge zur Geschichte des deutschen Verlagswesens. Festschrift für Heinz Sarkowski zum 65. Geburtstag, hrsg. v. M. Estermann und M. Knoche, Harrassowitz, Wiesbaden, S.158-176. Ders. (1991), Die Bergson-Rezeption in Deutschland, «Zeitschrift für philosophische Forschung», Bd. 45. 2., S. 257-266.

${ }^{16}$ Obwohl keine der beiden Sprachen seine Muttersprache ist, widmet sich Benrubi mit übermenschlicher Hingabe der Übersetzung. Dazu vgl. Benrubi, Isaak (1942), Souvenirs sur 
allerdings zum Aufschrei. "Das früheste große Werk von Bergson, 'Matière et mémoire', war zunächst in einer so unglaublich schlechten Übersetzung erschienen, daß es überhaupt nicht verstanden werden konnte" - so Simmels Sohn Hans in seinen Lebenserinnerungen ${ }^{17}$. Aus Bergsons Brief an Benrubi vom 3. März 1909, der für die Weiterführung der Übersetzungsarbeit prägend war, geht hervor, dass Simmel mit Diederichs und Bergson kurz darauf Kontakt aufgenommen haben muss. "J'ai reçu, il y a déjà une dizaine de jours, une lettre de M. Diederichs, me disant qu'il allait écrire à M. Simmel. Il devait d'ailleurs être déjà au courant de toute cette affaire avant que je lui eusse écrit, car, bien que je n'eusse pas mentionné dans ma lettre l'opinion de Simmel sur la traduction de 'Matière et mémoire', c'est sur ce point que sa réponse porte à peu près exclusivement [...]. Ne vous tourmentez pas au sujet de cette affaire, et croyez, je vous prie, à mes sentiments dévoués" ${ }^{18}$. Bergsons Werke sollten in einer besseren Übersetzung erscheinen als Matière et mémoire. So übernahm Simmel die Leitung der Übersetzungsarbeit und vertraute sie seinen zwei Schülerinnen Margarete Susman und Gertrud Kantorowicz an.

Die philosophische Anthropologie lässt sich nicht mehr auf den neuzeitlichen Begriff der Individualität, sondern nur auf der Grundlage einer Gesellschaftstheorie begründen - so Simmels Soziologie ${ }^{19}$. In Folge der Sozialdifferenzierung steigt der Freiheitsgrad moderner Individuen gegenüber einzelnen Bindungen bei gleichzeitiger Stärkung ihrer Abhängigkeit von der Summe der sozialen Beziehungen ${ }^{20}$. Die Theorie der Persönlichkeit muss vom

Henri Bergson, Neuchatel-Paris, Delachaux \& Niestlé. Bergsons Prosa ins Deutsche zu wiedergeben, überstieg allerdings Benrubis Kompetenz als Übersetzer. Anders ging es ihm bei Boutroux. Denn er konnte problemlos zwei seiner Werke ins Deutsche übersetzen: Boutrox, Émile (1907), Über den Begriff des Naturgesetzes, dt. von I. Benrubi, Jena, Diederichs. Ders. (1911), Die Kontingenz der Naturgesetze, dt. von I. Benrubi, Jena, Diederichs.

17 Hans Simmel, Lebenserinnerungen, Manuskript, 1 August 1941, Bielefeld, Simmelsarchiv, S. 59.

${ }^{18}$ Henri Bergson, Brief an Isaak Benrubi vom 3.3.1909, Fonds Isaak Benrubi, Bibliothèque publique et universitaire, Genève.

19 Simmel, Georg (1908), Soziologie. Untersuchung über die Formen der Vergesellschaftung, jetzt in Ders., GSG 11, hrsg. v. Otthein Rammstedt, Frankfurt/M, Suhrkamp, 1992, hier mit Soz zitiert, S. 13-14.

${ }^{20}$ Simmel Georg (1890), Über sociale Differenzierung. Soziologische und psychologische Untersuchungen, jetzt in GSG 2, Frankfurt/M, Suhrkamp, 1989, S. 109-295. 
Befund des fragmentarischen Charakters der Lebenserfahrung ausgehen ${ }^{21}$. Denn zwei entgegengesetzte Strömungen des Bewusstseins müssen dabei zum Einklang gebracht werden: einerseits das Leben des Menschen "für sich" und andererseits das Leben des Menschen für die sozialen Rollen, die er in der sozialen Wechselwirkung wahrnimmt. Ihre Synthese stellt die Bedingung der Möglichkeit dar, das Individuum in seinen sozialen Beziehungen $\mathrm{zu}$ sozialisieren. Diese Dialektik macht das “dritte Apriori” der Soziologie aus ${ }^{22}$. Das Misslingen der Synthese führt zur Entfremdung. Darin sieht Simmel das zentrale Problem der Moderne, dessen Lösung er einer Ethik der individuellen Lebensform anheimstellt ${ }^{23}$. Die rein pessimistische Betrachtung des modernen Persönlichkeitsproblems eint indessen Marx und die konservative Kulturkritik. Bergsons Metaphysik scheint ihnen Recht zu geben.

\section{Zeitlichkeit und Kinematographie}

Die Philosophie der durée stellt einen der wichtigsten Versuche dar, Kants Bewusstseinsphilosophie zu überwinden. Was Bergson als seine größte Entdeckung betrachtet, ist der Nachweis der Kontingenz des Bewusstseinsflusses, wie sie in der erlebten Zeitlichkeit unmittelbar anzutreffen ist. Die physisch-mathematische Zeitkategorie, auf der Kants transzendentale Ästhetik gründet, bezieht sich nicht auf das innere Zeitbewusstsein. Sein stetes Fließen ohne logisch-räumliche Strukturierung seiner Zustände lässt sich damit nicht erfassen. So besteht die Fehleinschätzung der Psychophysik darin, dass sie die erlebte Zeitlichkeit mit dem räumlichen Wahrnehmungsschema der Außenwelt abzubilden versucht ${ }^{24}$. Die Widerlegung der Psychophysik gilt Bergson jedoch als Mittel, um durch

${ }^{21}$ Simmel, Georg (1916/17), Der Fragmentcharakter des Lebens. Aus den Vorstudien zu einer Metaphysik, jetzt in Ders., GSG 13, Frankfurt/M, Suhrkamp, 2000, S. 202-216.

22 Dieser Umstand ist als die Erkenntnistheoretische Voraussetzung des Vergesellschaftungsprozesses zu betrachten und gilt Simmel in freier Anlehnung an Kants Kritik der reinen Vernunft als "transzendentale Voraussetzung" für das Bestehen der Erfahrungssphäre, deren Gegenstände die sozialen Beziehungsformen sind. Dazu vgl. Simmel, Georg (1908), Soziologie. Untersuchuneng über die Formen der Vergesellschaftung, jetzt in Ders., GSG 11, hrsg. v. Otthein Rammstedt, Frankfurt/M: Suhrkamp, 1992, hier mit Soz zitiert, S. 47-59.

${ }^{23}$ Simmel, Georg (1913), Das individuelle Gesetz. Ein Versuch über das Prinzip der Ethik, jetzt in Ders., (2001) GSG 12, Frankfurt/M., Suhrkamp, S. 417-470.

${ }^{24} \mathrm{Vgl}$. ED 7; ZF 7-8. 
die Kritik der naturwissenschaftlichen Methode Kants Bewusstseinsphilosophie an ihrer empfindlichsten Stelle infrage zu stellen: in der Lehre des Schematismus ${ }^{25}$. Indem die Möglichkeit einer psychophysischen Abmessung der Bewusstseinsinhalte widerlegt ist, wird auch das Zusammengehen der transzendentalen Ästhetik mit der transzendentalen Logik fraglich.

Der Raum ist laut Bergson ein Geistesakt, der die Wahrnehmung für die Zwecke des praktischen Handelns organisiert. Aus dem gattungsspezifischen Adaptionsprozess des Menschen geht die Trennung der Erfahrung in zwei Reiche hervor. Die räumliche Darstellung der Bewusstseinsinhalte folgt der Strukturierung der Außenwelt als "Handlungsfeld", dem gegenüber der heterogene Fluss der tiefen Qualitäten des Erlebnisses steht. Trotz der grundlegenden Bedeutung der durée für die Lebenserfahrung unterliegt sie der zersetzenden Wirkung der Raumformen. Die Wechselbeziehung des Bewusstseins mit der Außenwelt führt zur fortschreitenden "Kolonisierung" der Innenwelt durch den Raum. So zeigt Bergson wie sich das Bewusstsein durch die Berührung mit der Logik der Außenwelt umwandelt und die qualitative Mannigfaltigkeit der durée verdrängt. Die symbolische Darstellung des Bewusstseinsflusses in räumlichen Formen verwundert jedoch umso mehr, je tiefer die Betrachtung in das Leben des inneren Ich eindringt ( $E D 93$ f.; $Z F 98$ f.). Seine Tiefe und Oberfläche scheinen im gleichen Takt zu "schlagen" und lassen sich unter den übergreifenden Begriff der mathematisierten Zeit bringen. Unter der Oberfläche lebt jedoch ein magmatisches Tiefen-Ich weiter, obwohl sich die Menschen mit dessen Schatten zufrieden geben, das ihr praktisches Leben steuert ${ }^{26}$.

Das Bedürfnis, äußere Gegenstände sowie innere Zustände in die diskrete Reihenfolge der mathematischen Zeitachse einzuordnen, ist laut Bergson durch das soziale Leben bestimmt, dem sich das fragmentarische Ich der äußeren Erfahrung anpasst. So liegt der letzte Grund, um den zeitlichen Bewegungscharakter des Daseins zu verneinen, in der praktischen Struktur der Wahrnehmung. Sowohl die Intelligenz als auch die Sinne beschränken sich darauf, "vom Werden der Materie momentane und eben dadurch unbewegte

${ }^{25}$ Dazu vgl. Philonenko, Alexis (1994), Henri Bergson. Ou de la philosophie comme science rigoureuse, Paris, Cerf, S. 24. Vgl. auch Cohen, Hermann (1902), Logik der reinen Erkenntnis, Berlin, Cassirer.

${ }^{26}$ Für einen Vergleich zwischen Bergson und Freud vgl. Hum, Pierre (2007), L'oubli, 1880-1900, Lille, ANRT. 
Ansichten Punkt für Punkt aufzunehmen" (SE 276; EC 273). Dies macht den "kinematographischen Mechanismus" des Bewusstseins aus. Aus dem kontinuierlichen Fluss des Lebens nehmen die Menschen nur Momentbilder sub specie aeternitatis war. Es ist die "bedeutendste Illusion" der Erfahrung, die auf dem Glauben gründet, dass sich das Bewegte durch das Unbewegte rekonstruieren und denken lässt. Die Erkenntnistheorie darf darin nicht aufgehen, ohne die "letzte Wirklichkeit" der Welt - mithin die erlebte Zeitlichkeit ihres Wandels - aufzugeben.

Bergson strebt das Ziel an, die erlebte Zeitlichkeit in ihrer Unabhängigkeit von der praktisch-adaptiven Gestaltung der Erfahrung nachzuweisen. Dafür muss die praktische Wendung des Erkenntnisvermögens aufgehoben werden, indem sich das Bewusstsein daran gewöhnt, das Sein "unmittelbar" zu denken. Es muss versuchen zu "sehen, um zu sehen, nicht mehr zu sehen, um zu handeln" ( $S E$ 302; EC 298). Mit einem intuitiven Schritt lässt sich die "authentische Dimension" des Bewusstseins erschließen. So enthüllt "sich uns das Absolute in nächster Nähe, ja in gewissem Sinn in uns. Es ist psychologischen Wesens, nicht mathematischer oder logischer Art. Es lebt mit uns. Wie wir, nur in unendlich konzentrierter, in sich gesammelter Form, ist es Dauer" (ebd.). Am Wendepunkt seiner Argumentation angelangt, fragt sich Bergson, ob sich die "wahre Dauer" denkend beschreiben lässt, oder nur durch die kontemplative Anschauung ihres Fließens zu erfassen ist. Erst der Nachweis, dass sich die intuitive Wahrnehmung der erlebten Zeitlichkeit reflexiv fortsetzen lässt, ermöglicht zum letzten Kern der Lebensphilosophie vorzudringen. Eine Bedingung dieses Schritts ist, dass sich das Bewusstsein in den Fluss der durée "denkend" hineinversetzt, was die praktisch-adaptive Einstellung der Intelligenz nicht zulässt. So wird laut Bergson eine Kritik des philosophischen Formbegriffs notwendig. Die Funktion der Wahrnehmungsorgane ist durch die Adaption an die Umwelt bedingt und teilt die Welt in Qualitäten, Körper und Formen ein. Unter der Leitung des "kinematographischen Mechanismus" der Wahrnehmung werden dauerhafte Ansichten aus dem steten Wandel des Lebens ausgeschnitten, die "als Formen" betrachtet werden. Veränderung und Wandel lassen sich dann nur erkennen, als sie makroskopische Züge annehmen. "In Wirklichkeit jedoch ändert der Körper seine Form in jedem Augenblick. Oder besser, es gibt keine Form, da Form ein Unbewegtes ist, Wirklichkeit aber Bewegung. Real ist einzig die kontinuierliche Formveränderung; Form ist nur eine von einem Sich-Wandeln genommene Momentanaufnahme" (SE 305; EC 302).

Der Kunstgriff der Wahrnehmung zur praktischen Handhabe der stets im Wandel begriffenen Wirklichkeit besteht darin, mit einer abstrakten 
Vorstellung des Werdens zu operieren. "Diese Mischung aus einem eigenartigen und bestimmt umgrenzten Zustand mit der unbestimmten Veränderung überhaupt, ist es, die wir an Stelle der Eigenart der Veränderung setzen" (SE 308; EC 304). So bedient sich die Intelligenz desselben Verfahrens des Kinematographen, das die technische Übertragung der grundlegenden Wahrnehmungsstruktur in einen technischen Mechanismus darstellt. Indem der Intellekt das Werden künstlich rekonstruiert, widmet er sich nur der Oberfläche der Dinge, statt sich ihrem inneren Wesen hinzugeben (SE 309; $E C$ 305). Der Bewegungscharakter der Wirklichkeit lässt sich aber nur vermittelst des "Erkenntnisvermögens der Sympathie" erfassen. Methodologisch setzt dies voraus, dass sich die Erkenntnistheorie vom kinematographischen Mechanismus des Denkens verabschiedet. Es gilt, die Gegenstände nicht auf die Ideen zurückzuführen, sondern die statischen Formen in ihren Bewegungszusammenhängen aufzulösen. Mit diesem Ergebnis schließt Bergson den Kreis, der von seiner frühen Studie der durée über die Analyse der Ausdehnung in Matière et mémoire bis zur Évolution créatrice führt. Der Raum, die Zeit und die Kategorien des Intellekts lassen sich in ihrem "intuitiven Wahrheitsgehalt" auflösen, so dass sich Kants Lehre der Erfahrung überwinden lässt. Bergsons Kritik des Formbegriffs beschränkt sich allerdings auf die "verräumlichende Funktion" des Wahrnehmungsmechanismus und lässt keinen Formgebungsbegriff als Funktion der Erfahrungsbildung zu. Letzterer stand indessen im Zentrum der deutschen Debatte um die Ausdehnung des kantschen Ansatzes auf die Gesamtheit der Kulturwissenschaften.

\section{Formgebung und Konflikt}

Die Kritik des Formbegriffs und die Behauptung des Ursprungscharakters letzter Erfahrung der durée stellen die zwei Themen dar, an denen sich Simmel am deutlichsten von Bergson absetzt. Ist für Bergson eine intuitive Erkenntnis der Welt jenseits der Grenzen der Adaption möglich, so ist dies für Simmel ausgeschlossen. Erfahrung kommt nur durch die Wechselwirkung von Anschauung und Verstand zustande. Darin bleibt Simmel Kantianer: dem Menschen stehen keine monokausalen Erfahrungswege jenseits der Wechselwirkung zwischen der verbindenden Aktivität des Geistes und der sinnlichen Wahrnehmung zur Verfügung. So lässt sich auch die "Philosophie 
des Lebens" erst im Sinne Diltheys begründen ${ }^{27}$. Die "menschliche Erfahrungsstellung" ist in der Wechselwirkung ihrer Konstituenten zu erfassen. So lehnt Simmel die recherche du temps perdu als Vorstellung einer empathischen Apperzeption der durée ab, da sich ihm diese als eine Formgebungsmodalität des Bewusstseinsflusses unter anderen erschlie $3 \mathrm{t}^{28}$. Er betrachtet den Menschen als ein Lebewesen, das darauf angewiesen ist, sich selbst, seinem Bewusstseinsfluss und seiner Welt eine Form zu geben. Dies zeigt die Untersuchung des dynamischen Formbegriffs der "Grenze" in der Lebensanschauung ${ }^{29}$. Sie beharrt auf den Doppelaspekt des Lebens, gleichzeitig Selbstüberwindungsfluss (mehr Leben) und Fixierung durch Formgebung (mehr als Leben) zu sein. Letztere erfolgt in kognitiver, ästhetischer sowie gefühlsmäßiger Richtung und hindert das Bewusstsein daran, sich selbst "jenseits ihrer" zu erfahren.

Simmel übernimmt wichtige Aspekte von Bergsons Zeit- und Lebensphilosophie. Vor allem was die "Ethik der individuellen Lebensform" betrifft, ist die Vorstellung des aktuellen Lebens als Verdichtung seines vergangenen Zeitablaufs ausschlaggebend ${ }^{30}$. Trotzdem unterscheiden sich beide theoretischen Unterfangen grundlegend voneinander. Simmels Interesse konzentriert sich auf den Konflikt zwischen Leben und Formen als konstituierendes Merkmal sowohl des individuellen als auch des kollektiven Lebens der Menschen. Aus dieser Perspektive gilt das Leben, wie es Bergson umschreibt, lediglich als Teilphänomen des sich aus dem Konflikt zwischen Leben und Formen ergebenden, übergreifenden "Lebensprozesses". Die kontextuelle Untersuchung der geistes- und naturwissenschaftlichen "Grenzen des Lebens" dient der Erkenntnis der einheitlichen Erfahrungsstellung des Menschen. Sie setzt sich aus der kulturellen Formgebung des Ausdrucks, aus der organischen Umformung des Lebens durch den Lebens- und Sterbeprozess sowie aus der ethischen Gestaltung

27 Dilthey, Wilhelm (1883), Einleitung in die Geisteswissenschaften. Versuch einer Grundlegung für das Studium der Gesellschaft und der Geschichte, Erster Band, in Ders. (1959), Gesammelte Schriften, Bd. I., Stuttgart, Teubner.

${ }^{28}$ In der Untersuchung des Begriffs des Zustands kommt Simmel darauf zu sprechen. Dazu vgl. Simmel, Georg, Die historische Formung, Logos, Bd. 7. 1917/18, S. 113-152, S. 142 Fußnote.

${ }^{29}$ Simmel, Georg (1918), Lebensanschauung. Vier metaphysische Kapitel, Duncker und Humblot, München und Leipzig, jetzt in GSG 16, hrsg. v. Gregor Fitzi und Otthein Rammstedt, Suhrkamp, Frankfurt a.M., 1999, hier zitiert mit LBA, S. 209-425, S. 212 ff.

${ }^{30}$ Vgl. LBA, S. $346-425$. 
durch das individuelle Gesetz zusammen. Unter der Überschrift der "Transzendenz des Lebens" hebt Simmel das Aufeinander-Angewiesen-Sein des vitalen Lebensflusses mit der Formgebung hervor. "Transzendenz" bezeichnet hier den Umstand, dass das Leben in allen Existenzformen (von der Zelle über die unterschiedlichen Stufen des Organischen bis zum menschlichen Bewusstsein, den sozialen Beziehungen und den Kultursystemen) durch einen widersprüchlichen Prozess der Selbstüberwindung bestimmt ist. Er tendiert dazu, die aktuelle, lebenssichernde Form "als Grenze des Lebensflusses" zu überwinden (LBA 212 ff.). Dies dient ausschließlich der Erlangung einer neuen Formgebung, ohne dass sich der Komplexitätsgrad des Prozesses auf ein dialektisches Deutungsschema reduzieren lässt. Aus der Dynamik zwischen Leben und Formen gehen die unterschiedlichen Sphären der Erfahrung hervor, die sich zu autonomen Kultursphären mit einer eigenständigen Logik verdichten. In Folge dieser "Achsendrehung des Lebens" muss sich das schöpferische Leben ihrem Formgebungsdiktat beugen (LBA 236-296).

Im Bergsonismus spiegelt sich der Zeitgeist der Jahrzehnte vor dem Ersten Weltkrieg wider: les années électriques ${ }^{31}$. Es ist eine Krisenzeit, in der die empfundene Verflüssigung aller Lebensordnungen auf die Starrheit einer sich selbst aufgebenden Moderne prallt. Durch die Beschleunigung der Lebensrhythmen gelangt der Bruch der "menschlichen Lebensstellung" zu Bewusstsein. Simmel ist diese Erkenntnis bereits vor der Begegnung mit Bergsons Philosophie geläufig, wie seine Studie zur Großstadt bezeugt ${ }^{32}$. Ihre theoretische Verdichtung erfolgt jedoch in wesentlichen Zügen aus der Auseinandersetzung mit der sozialen Erscheinung des Konflikts ${ }^{33}$. In diesem Zusammenhang entwickelt sich Simmels lebensphilosophische Sichtweise aus einer frühen Intuition über die conditio humana, die ihm dank seiner humanistischen Bildung bewusst wird.

Beziehungen der Über- und Unterordnung, der Geheimhaltung und Schließung der sozialen Gruppe, der Treue und Dankbarkeit, aber auch die

${ }^{31}$ Prochasson, Christophe (1991), Les années électriques (1880-1910), La Decouverte, Paris.

32 Simmel, Georg (1903), Die Großstädte und das Geistesleben, jetzt in GSG 7, Frankfurt/M, Suhrkamp, 1995, S. 116-131.

${ }^{33}$ Simmel, Georg (1908), Der Streit, in Ders., Soziologie. Untersuchungen über die Formen der Vergesellschaftung (1908), jetzt in Ders. (1992), Georg Simmel Gesamtausgabe, Bd. 11, hrsg. v. Otthein Rammstedt, Suhrkamp, Frankfurt a.M., hier mit Soz zitiert, S. 284-382. 
"Ausschlussbeziehungen", die die Figuren des Armen und des Fremden verkörpern, stellen gleichwertige Modalitäten der Vergesellschaftung dar. Es gilt sie aus den erkenntnistheoretischen Prämissen der "Apriori der Soziologie" heraus zu erklären (Soz 47 ff.). Simmels Methodologie blendet die gemeinsame oder unterschiedliche Wertorientierung aus, die das soziale Handeln steuert, um unabhängig davon die Form zu untersuchen, die der Vergesellschaftungsprozess annimmt. An jedem Kapitel der Soziologie lässt sich die Theorie der Vergesellschaftung exemplifizieren. Die Konflikttheorie ermöglicht sie jedoch aus dem aktiven Widerspruch der Interessenlagen und Wertorientierungen programmatisch zu erklären (Soz 284-382). Ihre These lautet: gerade weil Menschen und soziale Gruppen sich im Konflikt befinden, integrieren sie sich in ein übergreifendes soziales Geflecht.

Bereits zu Simmels Lebenszeit bestand ein wissenschaftlicher Grundkonsens darüber, dass der Kampfbegriff von soziologischer Bedeutung ist: die Tatsache des Konfliktes fördert Interessengemeinschaft und Organisation auf jeder Konfliktseite. Schon deshalb ist der Kampf als Träger von Vergesellschaftung zu sehen. Darüber hinaus stellt sich für die Konfliktsoziologie jedoch die Frage, ob der Kampf, mithin die Situation, in der die Mitbeteiligten explizit gegeneinander handeln, eine Vergesellschaftungsform darstellt. Simmels Ansatz geht von einem analytischen Gesichtspunkt aus und rekonstruiert schrittweise die Bausteine, die die Frage sozialtheoretisch bejahen lassen. Als "dissoziierend" gelten die Ursachen des Kampfes, wobei sich der Kampf selbst als "Abhilfsbewegung" deuten lässt, um gegen den "auseinanderführenden Dualismus" anzutreten (Soz 284). Man kämpft gegeneinander und hält damit die soziale Wechselwirkung aufrecht, die durch Kampfverweigerung auseinanderbrechen würde. Es handelt sich dabei um eine Spielart des "gemeinsamen Handelns" ohne gemeinsame Wertorientierung. Herkömmlich geht die Sozialphilosophie davon aus, dass die Einheit der sozialen Gruppe nach dem Ausbruch des Kampfes lediglich durch Versöhnung oder durch die Vernichtung einer der Konfliktparteien wiederherzustellen ist. Demgegenüber bietet der Konflikt einen dritten Weg, auf dem die Einheit der sozialen Beziehung "als Kampf" beibehalten wird. Der "Wille zum Konflikt" bedeutet allerdings nicht die Trivialität eines si vis pacem para bellum, da jenseits der Absicht der Handelnden der Kampf selbst schon eine Auflösung der absoluten in einer wechselwirkenden Spannung der Gegensätze bedeutet. Der Kampf klärt und gestaltet die sozialen Beziehungen, indem er latente Konflikte innerhalb der Gruppe expliziert und in Wechselwirkungsformen umwandelt. Umgekehrt bedeutet die Ablehnung des Kampfes nicht "Gleichgültigkeit", da sie zur 
Abschwächung des Vergesellschaftungsprozesses und in letzter Konsequenz zu seiner Auflösung führt. Zwei Entwicklungen, die an sich "Negationen" sind. Das positive Moment des Kampfes liegt indessen darin, dass er die Kämpfenden in der sozialen Form des "Gegeneinander-Handelns" vereinigt.

Die Argumentation zum positiven Ertrag des Konflikts als soziale Wechselwirkungsform lässt sich als Kritik von Tönnies soziologischem Ansatz lesen ${ }^{34}$, da für Simmel "die herkömmlichen Gegenstände der Soziologie nur eine Unterabteilung dieser weitgreifenden, wirklich von einem Prinzip bestimmten Wissenschaft bilden" (Soz 285). Sie gründet auf der formalen Betrachtung der sozialen Wechselwirkungen, die sowohl ihre bejahende als auch ihre verneinende Spielart berücksichtigt. Methodologisch vernachlässigt die Soziologie die soziale Erscheinung des Kampfes, solange sie sich auf den Einheitsgegenstand "Gesellschaft" konzentriert. Wenn ihre Einheit jedoch als Zusammensetzung von Vergesellschaftungsprozessen betrachtet wird, gebührt dem Kampf eine zentrale Stellung im soziologischen Kategorienfeld. Parallel dazu, wie die Fragestellung des dritten Apriori der Soziologie hervorhebt, ist auch die "Einheit" der vergesellschafteten Individualität als Ergebnis eines Kampfes zu verstehen (Soz 59 f.). Weder das Individuum noch die Gemeinschaft oder die Gesellschaft als Spielarten der sozialen Gruppe lassen sich restlos als harmonische Einheiten darstellen. "Wie der Einzelne die Einheit seiner Persönlichkeit doch nicht nur so gewinnt, daß ihre Inhalte nach logischen oder sachlichen, religiösen oder ethischen Normen restlos harmonieren, sondern wie Widerspruch und Streit nicht nur dieser Einheit vorangehen, sondern in jedem Augenblick ihres Lebens in ihr wirksam sind - so dürfte es keine soziale Einheit geben, in der die konvergierenden Richtungen der Elemente nicht von divergierenden unablöslich durchzogen wären. Eine Gruppe die schlechthin zentripetal und harmonisch, bloß 'Vereinigung' wäre, ist nicht nur empirisch unwirklich, sondern sie würde auch keinen eigentlichen Lebensprozeß aufweisen" (ebd.).

Auf dieser Ebene der Argumentation angelangt, tritt Simmels humanistische Bildung auf den Plan ${ }^{35}$. Sie konterkariert die Feststellung der

34 Tönnies, Ferdinand (1887), Gemeinschaft und Gesellschaft. Abhandlung des Communismus und des Sozialismus als empirische Kulturformen, Leipzig, Reisland. Jetzt: Ders., (1988). Gemeinschaft und Gesellschaft. Grundbegriffe der reinen Soziologie, Darmstadt, Wissenschaftliche Buchgesellschaft, (Neudr. d. 8. Aufl. von 1935, 2. unveränd. Aufl.), S. 3.

${ }_{35}$ Simmel, Georg (1884), Dantes Psychologie, jetzt in GSG 1, hrsg. v. Klaus Christian Köhnke, Frankfurt/M, Suhrkamp, 1999, S. 91-177. 
Unlebendigkeit rein harmonischer Vereinigung mit zwei Beobachtungen, die es ermöglichen, sie auf die übrigen Kultursphären auszudehnen und somit den Grundstein einer lebensphilosophischen Konzeption zu legen. Simmel geht von der Kritik der Theorie harmonischer sozialer Verbindung aus: "Die Gesellschaft der Heiligen, die Dante in der Rose des Paradieses erblickt, mag sich so verhalten, aber sie ist auch jeder Veränderung und Entwicklung enthoben, während schon die heilige Versammlung der Kirchenväter in Raphaels Disputa sich, wenn nicht als wirklicher Streit, so doch als eine erhebliche Verschiedenheit von Stimmungen und Denkrichtungen darstellt, aus der die ganze Lebendigkeit und der wirkliche, organische Zusammenhang jenes Zusammenseins quillt" (Soz 285 f.). Rückblickend aus der Perspektive von Plessners Kritik der Gemeinschaft ${ }^{36}$, lässt sich hier beobachten, inwiefern Simmel auf eine "zeitliche Strukturgrenze der Gemeinschaft" im Sinne einer lebendigen sozialen Verbindung verweist, die mit der Konfliktdynamik der sozialen Gruppe zusammenhängt. Eine vollständig harmonische und konfliktlose Vergesellschaftung würde zu einem Stillstand im "Lebensprozess" der sozialen Gruppe führen. Wie der Kosmos-Begriff der antiken Philosophie Liebe und Hass, attraktive und repulsive Kräfte voraussetzt, die ihn als solchen ermöglichen, so braucht die moderne Gesellschaft ein bestimmtes Verhältnis von Harmonie und Disharmonie, Assoziation und Konkurrenz, um fortzubestehen. All diese Entzweiungserscheinungen sind keineswegs bloß "soziologische Passiva", negative Instanzen, als sei die Gesellschaft nur von Tönnies' "bejahenden Verbindungen" zusammengehalten (Soz 286).

Laut Simmel ist jedoch nicht ausreichend festzustellen, dass eine auf die bejahenden Beziehungen begrenzte Soziologie wie die von Ferdinand Tönnies fehlschlagen muss. Vielmehr geht es darum, kritisch zu rekonstruieren, aus welcher Begriffsvermengung die Einschränkung der Soziologie auf die "positiven Wechselwirkungsformen" hervorgeht. Das Missverständnis entspringt aus dem Doppelsinn des Einheitsbegriffs in der vorwissenschaftlichen Sprache. Denn als "Einheit" bezeichnet man einerseits die Übereinstimmung im Gegensatz zur Entzweiung und betritt dadurch den Plan einer normativen Betrachtung der Fragestellung. Andererseits heißt

36 Plessner, Helmuth (1924), Die Grenzen der Gemeinschaft. Eine Kritik des sozialen Radikalismus. Bonn, Cohen. Jetzt in Ders. (1981), Gesammelte Schriften, Bd. 5. Macht und Menschliche Natur, Frankfurt/M, Suhrkamp, S. 7-133. 
"Einheit" aber auch Gesamtsynthese von "Personen, Energien und Formen zu einer Gruppe" in einem rein deskriptiven Sinn (Soz 287). Damit ist die Ganzheit ihrer sowohl einheitlichen wie dualistischen Beziehungen gemeint, jenseits von jeder Form der Eintracht. Der logische Fehlschluss ergibt sich daraus, dass bestimmte Charakteristika der sozialen Gruppe als Ganzes aus den gleichen Eigenschaften ihrer Teile abgeleitet werden. "So führen wir das Gruppengebilde, das wir als 'einheitlich' empfinden, insoweit auf diejenigen seiner funktionellen Bestandteile zurück, die als die spezifisch einheitlichen also mit Ausschluß der andern, weiteren Bedeutung des Wortes - gelten" (ebd.). Zur Ungenauigkeit trägt in umgekehrter Betrachtungsrichtung auch der Doppelsinn der Begriffe von Entzweiung und Opposition bei. Indem sie ihren verneinenden und zerstörerischen Sinn zwischen den einzelnen Elementen entfalten, wird daraus unbefangen geschlossen, dass sie sich in derselben Weise auch auf das gesamte Verhältnis auswirken. Negation zwischen den Teilen bedeutet jedoch nicht automatisch Negation des Ganzen, wie auch die Konkurrenzbeziehungen auf dem wirtschaftlichen Gebiet zeigen.

Die Generalisierung dieser Beobachtungen, die Simmel im Rahmen der Konfliktsoziologie macht, bietet den Ausgangspunkt für seine spätere Lebensphilosophie. So lässt sich zeigen, wie der Konfliktbegriff erst ausgehend von dieser theoretischen Reflexion die Bedeutung annimmt, die einen übergreifenden Lebensbegriff als wechselseitigen Überwindungsprozess von Leben und Formen aufzustellen ermöglicht. Erst auf dieser Grundlage lässt sich das Leben als steter Wachstumsfluss einerseits und als Spaltung in Leben und Formen andererseits denken, dessen Konflikt die Überwindung der jeweils vorläufigen Lebensform ermöglicht. In der Lebensanschauung dekliniert Simmel diese Beobachtung in den unterschiedlichen Richtungen seiner späten Reflexion durch. Das biologische Leben nimmt die Form der Zelle oder des Organismus an, die sich spalten oder reproduzieren und somit $\mathrm{zu}$ neuer Formgebung gelangen. Das Bewusstseinsleben wandelt von einer Synthese zur nächsten sowie sich die Kultursysteme vom existierenden zum neuentstehenden Stil entwickeln. Ähnliches gilt für die Wirtschaftsformen und die Sozialsysteme, obwohl die Formen hier dazu tendieren, sich zu verselbständigen und die Erneuerung des Lebens zu erschweren. So spitzt sich der Konflikt der Kultur bis zu ihrer Krise und zur konsequenten Ablehnung der Form als solche zu. Stellvertretend für die Lage der gesamten modernen Kultur an der Schwelle zum Ersten 
Weltkrieg bezeugt dies laut Simmel der Übergang der modernen Kunst vom Impressionismus zum Expressionismus ${ }^{37}$.

Gesellschaft resultiert sowohl aus bejahenden als auch aus verneinenden sozialen Beziehungen, wie Simmel Soziologie 1908 im Widerspruch zu Ferdinand Tönnies beobachtet. Dabei handelt es sich um den soziologischen Fall eines Gegensatzes "in der Lebensauffassung überhaupt" "38. Für die herkömmliche Betrachtung des Konfliktbegriffs stehen sich "allenthalben zwei Parteien des Lebens gegenüber, von denen die eine sein Positives, seinen eigentlichen Inhalt, oder sogar die Substanz des Lebens selbst trägt, die andre aber das seinem Sinn nach Nicht-Seiende ist" (Soz 286, Fußnote). Aus dualistischer Perspektive betrachtet, kommt das Positive des Lebens erst nach Abzug dieses Aspekts zur Geltung; so verhalten sich Glück und Leid, Tugend und Laster, Stärke und Unzulänglichkeit, Gelingen und Fehlschlagen, als unvereinbare Gegensätze zueinander. Aus der Konfliktsoziologie geht jedoch eine "dynamische Auffassung" der grundlegenden Widersprüchlichkeit von Welt und Leben hervor. "Als die höchste Auffassung indes, die diesen Gegensatzpaaren gegenüber angezeigt ist, erscheint mir die andre: alle diese polaren Differenziertheiten als ein Leben zu begreifen, auch in dem, was von einem einzelnen Ideal aus nicht sein soll und ein bloß Negatives ist, den Pulsschlag einer zentralen Lebendigkeit zu spüren, den Gesamtsinn unsrer Existenz aus beiden Parteien erwachsen zu lassen" (ebd.).

So trifft man hier in nuce auf Simmels Lebensanschauung. Im "umfassenden Zusammenhang" des Lebens übt auch das, was vereinzelt als störend und zerstörend empfunden wird, eine positive Wirkung aus, schlägt keinen Riss in das Lebensgewebe, sondern erfüllt eine nur ihm vorbehaltene Rolle. Abschließend lassen sich somit die Ergebnisse der konfliktsoziologischen Untersuchungen auf das Ganze des Lebens übertragen: "Nun mag die Höhe, von der aus alles, was in den sachlichen und den Wertreihen als Plus und Minus, als einander widersprechend, einander aufhebend sich entgegentritt, dennoch zu einer Lebenseinheit ineinander gefühlt wird - diese Höhe zu erreichen oder durchgängig festzuhalten, mag uns versagt sein; zu gern denken und empfinden wir unser wesentliches Sein, das, was wir eigentlich und im letzten Grunde bedeuten, als mit einer dieser

${ }^{37}$ Simmel, Georg (1918), Der Konflikt der modernen Kultur. Ein Vortrag, München und Leipzig, Duncker und Humblot, jetzt in GSG 16, S. 181-207.

${ }^{38}$ Vgl. Simmel, Georg (1908), Der Streit, in Ders., Soziologie. GSG 11 a.a.O., S. 284-383, bes. S. 286 f., Fußnote. 
Parteien identisch, je nach unsrem optimistischen oder pessimistischen Lebensgefühl erscheint uns die andre als Oberfläche, Zufälligkeit, als etwas, das zu beseitigen oder in Abzug zu bringen ist, damit sich das wahre und in sich einheitliche Leben ergäbe. In diesem Dualismus [...] sind wir allenthalben, von den engsten bis zu den umfassendsten Lebensprovinzen personaler, sachlicher, sozialer Art, verwickelt: daß wir eine Ganzheit oder Einheit haben oder sind, die in zwei logisch und sachlich entgegengesetzte Parteien auseinandergeht, und dass wir nun jene Totalität unser mit einer dieser Parteien identifizieren und die andre als ein Fremdes eigentlich nicht dazu Gehöriges und unser zentrales und umfassendes Wesen Verneinendes empfinden. Zwischen dieser Tendenz und der andern, die das Ganze auch wirklich Ganzes sein läßt, die die Einheit, die doch schließlich beide Gegensätze befaßt, auch wirklich in jedem von beiden und ihrem Zusammen lebendig macht - bewegt sich dauernd das Leben. Das Recht der letzteren Tendenz aber an der soziologischen Erscheinung des Streites zu behaupten, ist umso erforderlicher, als der Streit seine gesellschaftszerstörerische Kraft als eine scheinbar undiskutable Tatsache aufdrängt" (Soz 286 f., Fußnote).

Gegenüber Bergsons Appell, sich der durée hinzugeben, ist somit Simmel schon 1908 gewappnet. Die Aufgabe einer Anthropologie, die in der Lage ist die menschliche Erfahrungsstellung in ihrer natürlichen, kulturellen und sozialen Komplexität zu begründen, besteht darin, ihren dualistischen Charakter als Einheit zu betrachten. Die unterschiedlichen Ströme der Erfahrung - wie etwa Innenwelt, Außenwelt und Mitwelt - gegeneinander auszuspielen, bedeutet somit nicht die durch die Adaption bedingte menschliche Erfahrungsstellung zu überwinden, sondern sich in ihr gerade zu verfangen. Der vergesellschaftende Charakter moderner sozialer Wechselwirkung erhält sich trotz aller Konflikte zwischen seinen Bestandteilen. Dies gilt auch für die Individualität. Sie konstituiert sich und bestätigt sich als solche im alltäglichen Kampf um die soziologischen Apriori. Schafft sie es, den privaten und den öffentlich gewendeten Bewusstseinsfluss miteinander zu verbinden, ist sie sozialisiert und kann sich innerhalb ihrer sozialen Beziehungen verwirklichen. Wendet sie sich indessen lediglich der recherche du temps perdu zu, so ist sie bereits auf dem Wege der Intimisierung und gibt die Möglichkeit auf, die kulturelle Formgebung des sozialen Lebens für sich positiv zu wenden. 